\title{
Single-trial analyses: why bother?
}

\section{Cyril R. Pernet ${ }^{1}{ }^{*}$, Paul Sajda $^{2}$ and Guillaume A. Rousselet ${ }^{3}$}

\author{
Brain Research Imaging Centre, SINAPSE Collaboration, University of Edinburgh, Edinburgh, UK \\ 2 Department of Biomedical Engineering, Columbia University, New York, NY, USA \\ ${ }^{3}$ Centre for Cognitive Neuroimaging, Institute of Neuroscience and Psychology, University of Glasgow, Glasgow, UK \\ ${ }^{*}$ Correspondence: cyril.pernet@ed.ac.uk
}

Neuroimaging techniques have been traditionally used to demonstrate differences between means calculated across conditions or groups of subjects. However, as illustrated by the articles in this research topic, by studying the variability across trials, singletrial analyses can in some situations allow us to go beyond this kind of imaging to the mean. Indeed, single-trial analyses can provide additional information that is unobservable if we collapse the data to a mean. For example, single-trial analyses can help us provide a systematic mapping between (i) brain activity and stimulus information space (Schyns, 2010; Rousselet et al., 2011), (ii) brain activity and subject's behavioral variability (Ratcliff et al., 2009), and (iii) brain activity measured using different imaging techniques, e.g., fMRI and EEG (Goldman et al., 2009; deBettencourt et al., 2011). Importantly, using certain parametric experimental designs, single-trial analyses can give us access to brain mechanisms, by allowing us to specify the information content of brain activity and its transformation (Schyns, 2010; Rousselet and Pernet, 2011).

Single-trial analyses refer to methods that consider the variance within subjects. Two broad families of methods can be distinguished: univariate methods extract information among trials in space, time, or both; multivariate methods extract information across space, time, or both, in individual trials. Single-trial analyses may thus be used for behavioral experiments (e.g., Etchells et al., 2011) and neuroimaging experiments (e.g., Cohen and Cavanagh, 2011; Macdonald et al., 2011; Milne, 2011; Rousselet et al., 2011; Touryan et al., 2011; Wutte et al., 2011). Single-trial analyses of neuroimaging data have seen their use increase since the late 1960s, starting with Donchin (1969). Despite this long tradition and several advantages over group analyses, single-trial analyses remain nevertheless marginal.

The simplest form of univariate single-trial analysis is a regression over all of the trials in single subjects, to measure the relationship between, e.g., the signal amplitude and a parameterized stimulus space. This approach is often referred to as parametric design in fMRI. In this Research Topic, Rousselet et al. (2011) showed that a similar approach can be used in EEG to quantify brain responses to stimulus information in individual subjects, and characterize the probability of observing a mapping between stimulus information and EEG amplitude, thus going beyond the study of the average brain. Cohen and Cavanagh (2011) also demonstrated that the single-trial parametric approach can be extended to time-frequency decompositions of power and phase. Variance among trials also contains information about subjects or cognitive states. For instance, Milne (2011) established that children with autism have significantly more variance in the latency of their P1 response to Gabor patches than control participants. Macdonald et al. (2011) found that pre-stimulus alpha power is correlated with subjects' judgment of attentional state (see also VanRullen et al., 2011 for a review of evidence linking alpha oscillations to perception and attention).

Multivariate methods are often used to characterize the spatial-temporal variance in each trial in order to derive pattern classifiers (see however Friston et al., 1996 for a more traditional use). For instance, Touryan et al. (2011) used the variance in space and time to train a discriminant function to classify, in real time, brain activity related to familiar and unfamiliar faces. In their experiment, although the group ERPs differed between familiar and unfamiliar faces over frontal and parietal electrodes, the classification revealed that only the parietal response allowed the discrimination of the stimulus category on a single-trial basis. This result illustrates that group averaging may be misleading, presenting an abstract signal that cannot be found in individual subjects (see also Gaspar et al., 2011). Wutte et al. (2011) also used a pattern classification technique (support vector machine) to read-out motion direction from areas V1 and MT+ using fMRI. Although the spatial variance reflected the direction of perceived stimuli, individual perceptual thresholds were predicted by the relative variance in amplitude between activation and rest trials, thus illustrating the complementarity of univariate and multivariate methods.

In addition to a unique window on brain mechanisms, singletrial analyses also allow researchers to interpret individual differences by quantifying effects within and between subjects, providing a richer data description mandatory to build efficient models of perception and decision-making. It is often said that single-trial analyses require either too many trials, or dense coverage (dense arrays in MEEG or fast TR in fMRI), or both. It is true that in order to obtain good signal-to-noise ratio (regression over trials) many trials are necessary and, in order to obtain good patterns ("weighting" across electrodes/voxels, time intervals, frequency intervals), dense coverage is mandatory. Many trials are nevertheless also mandatory for an average to be a meaningful measure (Rousselet et al., 2008), just as dense coverage is necessary to ensure that minima or maxima located between sampled time points in fMRI or between channels in MEEG are not overlooked. There are a growing number of user friendly toolboxes available to perform single-trial analyses (e.g., Parra et al., 2005; Hanke et al., 2009; Delorme et al., 2011; Hartmann et al., 2011; Oostenveld et al., 2011; Pernet et al., 2011). We encourage everyone interested in understanding how the stimulus space and behavioral response map onto brain activity to use these tools rather than merely amass binary results showing group differences in brain activity among conditions (Rousselet and Pernet, 2011). 


\section{REFERENCES}

Cohen, M. X., and Cavanagh, J. F. (2011). Single-trial regression elucidates the role of prefrontal theta oscillations in response conflict. Front. Psychol. 2:30. doi: 10.3389/fpsyg.2011.00030

deBettencourt, M., Goldman, R., Brown, T., and Sajda, P. (2011). Adaptive thresholding for improving sensitivity in single-trial simultaneous EEG/fMRI. Front. Psychol. 2:91. doi: 10.3389/fpsyg.2011.00091

Delorme, A., Mullen, T., Kothe, C., AkalinAcar, Z., Bigdely-Shamlo, N., Vankov, A., and Makeig, S. (2011). EEGLAB, SIFT, NFT, BCILAB, and ERICA: new tools for advanced EEG processing. Comput. Intell. Neurosci. 2011, 130714.

Donchin, E. (1969). Discriminant analysis in average evoked response studies: the study of single-trial data. Electroencephalogr. Clin. Neurophysiol. 27, 311-314.

Etchells, P. J., Benton, C. P., Ludwig, C. J. H., and Gilchrist, I. D. (2011). Testing a simplified method for measuring velocity integration in saccades using a manipulation of target contrast. Front. Psychol. 2:115. doi: 10.3389/ fpsyg.2011.00115

Friston, K. J., Stephan, K. M., Heather, J. D., Frith, C. D., Ioannides, A. A., Liu, L. C., Rugg, M. D., Vieth, J., Keber, H., Hunter, K., and Frackowiak, R. S. J. (1996). A multivariate analysis of evoked responses in EEG and MEG data. Neuroimage 3, 167-174.
Gaspar, C.M., Rousselet, G.A., and Pernet, C. R. (2011). Reliability of ERP and single-trial analyses. Neuroimage 58, 620-629.

Goldman, R. I., Wei, C. Y., Philiastides, M. G., Gerson, A. D., Friedman, D., Brown, T. R., and Sajda, P. (2009). Single-trial discrimination for integrating simultaneous EEG and fMRI: identifying cortical areas contributing to trial-to-trial variability in the auditory oddball task. Neuroimage 47, 136-147.

Hanke, M., Halchenko, Y. O., Sederberg, P. B., Hanson, S. J., Haxby, J. V., and Pollmann, S. (2009). PyMVPA: a python toolbox for multivariate pattern analysis of fMRI data. Neuroinformatics 7, 37-53.

Hartmann, T., Schulz, H., and Weisz, N. (2011). Probing of brain states in real-time: introducing the console environment. Front. Psychol.2:36. doi: 10.3389/fpsyg.2011.00036

Macdonald, J. S. P., Mathan, S., and Yeung, N. (2011). Trial-by-trial variations in subjective attentional state are reflected in ongoing prestimulus EEG alpha oscillations. Front. Psychol. 2:82. doi: 10.3389/fpsyg.2011.00082

Milne, E. (2011). Increased intra-participant variability in children with autistic spectrum disorders: evidence from single-trial analysis of evoked EEG. Front. Psychol.2:51. doi: 10.3389/ fpsyg.2011.00051

Oostenveld, R., Fries, P., Maris, E., and Schoffelen, J. M. (2011). FieldTrip: open source software for advanced analysis of MEG, EEG, and invasive electrophysiological data. Comput. Intell Neurosci. 2011, 156869.

Parra, L. C., Spence, C. D., Gerson, A. D. and Sajda, P. (2005). Recipes for the linear analysis of EEG. Neuroimage 28, 326-341.

Pernet, C. R., Chauveau, N., Gaspar, C. and Rousselet, G. A. (2011). LIMO EEG: a toolbox for hierarchical linearmodeling of electroencephalographiuc data. Comput. Intell. Neurosci. 2011, 831409.

Ratcliff, R., Philiastides, M. G., and Sajda, P. (2009). Quality of evidence for perceptual decision making is indexed by trial-to-trial variability of the EEG. Proc. Natl. Acad. Sci. U.S.A 106, 6539-6544.

Rousselet, G. A., Gaspar, C. M. Wieczorek, K. P., and Pernet, C. R. (2011). Modeling single-trial ERP reveals modulation of bottom-up face visual processing by top-down task constraints (in some subjects). Front. Psychol. 2:137. doi: 10.3389/ fpsyg.2011.00137

Rousselet, G. A., Husk, J. S., Bennett, P. J., and Sekuler, A. B. (2008). Time course and robustness of ERP object and face differences. J. Vis. 8, 3.1-18.

Rousselet, G. A., and Pernet, C. R. (2011). Quantifying the time course of visual object processing using ERPs: it's time to up the game. Front. Psychol. 2:107. doi: 10.3389/ fpsyg.2011.00107

Schyns, P. G. (2010). Grand challenges in perception science: modeling the future. Front. Psychol. 1:10. doi: 10.3389/fpsyg.2010.00010

Touryan, J., Gibson, L., Horne, J. H., and Weber, P. (2011). Real-time measurement of face recognition in rapid serial visual presentation. Front. Psychol. 2:42. doi:10.3389/fpsyg.2011.00042

VanRullen, R., Busch, N. A., Drewes, J., and Dubois, J. (2011). Ongoing EEG phase as a trial-by-trial predictor of perceptual and attentional variability. Front. Psychol. 2:60. doi: 10.3389/ fpsyg.2011.00060

Wutte, M. G., Smith, M. T., Flanagin, V., and Wolbers, T. (2011). Physiological signal variability in hMT+ reflects performance on a direction discrimination task. Front. Psychol. 2:185. doi: 10.3389/fpsyg.2011.00185

Received: 17 August 2011; accepted: 20 October 2011; published online: 08 November 2011.

Citation: Pernet CR, Sajda P and Rousselet GA (2011) Single-trial analyses: why bother? Front. Psychology 2:322. doi: 10.3389/fpsyg.2011.00322

This article was submitted to Frontiers in Perception Science, a specialty of Frontiers in Psychology.

Copyright (C) 2011 Pernet, Sajda and Rousselet. This is an open-access article subject to a non-exclusive license between the authors and Frontiers Media $S A$, which permits use, distribution and reproduction in other forums, provided the original authors and source are credited and other Frontiers conditions are complied with. 